PACS 83.10.Rs, 63.22.Rc

\title{
Low frequency vibrations of carbon nanoscrolls
}

\author{
A.V. Savin ${ }^{1, \dagger}$, E.A. Korznikova ${ }^{2}$, S.V. Dmitriev ${ }^{2,3}$ \\ †asavin00@gmail.com
}

\begin{abstract}
${ }^{1}$ Semenov Institute of Chemical Physics, Russian Academy of Sciences, Kosygin St. 4, Moscow 117977, Russia
${ }^{2}$ Institute for Metals Superplasticity Problems, Russian Academy of Science, Khalturin St. 39, Ufa 450001, Russia

${ }^{3}$ Tomsk State University, Lenin Prospekt 36, Tomsk 634050, Russia
\end{abstract}

Graphene is a recently discovered material with prominent properties those zero flexural rigidity allows creation of different volume conformations. The preferable geometric configuration is determined by the balance of energy gain due to increase of the number of atoms involved in van der Waals interactions with the energy loss due to graphene bending. One can recall such common conformations as folds, scrolls and other secondary structures. Full atomistic modelling of long nanoribbon dynamics requires considerable simulation machines powers and in order to solve this problem a simple model of a molecular chain on the plane, which allows the description of folded and scrolled packages of graphene nanoribbons has been proposed. Earlier possible steady states of graphene nanoribbons have been obtained using this model and compares to full atomic modelling. In this paper the simulation of high-frequency nonlinear vibrations of scrolled carbon nanoribbons packages was performed. It was revealed that the first three low-frequency natural scroll vibrations ("twisting-untwisting" and "transverse compression" modes) are stable only if their energy does not exceed $0.1 \mathrm{eV}$, and the interaction of these modes starts to occur at higher energy. The excitation of one mode leads to the excitation of the two others.

Keywords: graphene nanoscrolls, natural vibrations, molecular dynamics.

\section{Introduction}

The carbon atoms are able to create a huge variety of structures, including recently discovered its monatomic layer - graphene attracting a great attention of researchers $[1,2,3,4,5,6,7]$. This $2 \mathrm{D}$ nanomaterial exhibits high values of electrical and thermal conductivity, rigidity and tensile strength, the enormous surface area and other properties, providing a wide variety of its potential applications. Flexural rigidity of graphene allows creation of folds and scrolls, those stability is provided by relatively weak van der Waals interaction between the carbon atoms. The geometric configuration is determined by the balance of energy gain due to increase of the number of atoms involved in van der Waals interactions with the energy loss due to graphene bending $[8,9]$.

The possibility of the formation of graphene rolls during the abrasion of the graphite lubricant has been revealed in 1960 [10]. A number of experimental methods for obtaining graphene nanoribbons scrolls and the study of their structure and properties has been developed up to date $[11,12,13$, $14,15,16,17]$. Besides experimental methods properties of graphene nanoscrolls are widely explores by means of $\mathrm{ab}$ initio calculations $[18,19,20]$, molecular dynamics $[21,22$, $23,24,25,26,27,28,29,30,31,32]$, molecular mechanics [33] and continual models of elastic spiral shaped rod [21, $26,34,35]$.

It has been already mentioned that the stability of the scroll structure is provided by weak nonvalent carbon atoms interactions. Due to this fact such structure types should possess a low frequency (gigahertz order) natural oscillations. The lowest frequency vibration corresponds to periodical twisting and untwisting of the scroll. The presence of this vibration type [21] provides the possibility of scroll application as an nanooscillator or nanoactuator in mechanical nanodevices. In this paper, we perform modelling and analysis of the low-frequency high-amplitude oscillations of nanoribbon scrolls, namely periodic twisting-untwisting mode and scroll transverse stretching-compression mode.

\section{Chain model of the nanoribbon}

Full-atomic models used for modelling carbon folds and scrolls until recently were found to be very demanding in computational power when considering long-term dynamics of large size nanoribbons configurations. Complexity of those models hampers the simulation results analysis. In order to overcome these obstacles authors of current paper have proposed a simple model of the planar molecular chain capable of describing the longitudinal and flexural motion of carbon nanoribbon and allowing the study of its volume conformations [36, 37]. Possible stationary configurations of nanoribbon scrolls, their energetical characteristics and natural vibrations frequency have been investigated by means of proposed model.

In this model the dynamics of the nanoribbon is reduced to the description of the dynamics of the molecular chain where every node corresponds to one layer of the cross section of the considered nanoribbon. A nanoribbon scroll configuration in this case corresponds to spiral shape of the molecular chain packing in 2D space - see Fig. 1. 
Graphene nanoribbon is a narrow, straight-edged stripe of graphene. The detailed description of the chain model allowing to analyse the folded and rolled packaging of long graphene nanoribbons can be found in [36, 37].

The following Hamiltonian can describe the chain model of carbon nanoribbon

$$
\begin{aligned}
H= & \sum_{n=1}^{N} \frac{1}{2} M\left(\dot{x}_{n}^{2}+\dot{y}_{n}^{2}\right)+\sum_{n=1}^{N-1} V\left(r_{n}\right)+ \\
& +\sum_{n=2}^{N-1} U\left(\theta_{n}\right)+\sum_{n=1}^{N-4} \sum_{k=n+4}^{N} W\left(r_{n, k}\right),
\end{aligned}
$$

where the vector $u_{n}=\left(x_{n}, y_{n}\right)$ sets the coordinates of $n$-th particle, number of nodes $N$ establishes the nanoribbon length $L=(N-1) a, M=12 m_{p}$ is the mass of carbon atom $\left(m_{p}=1.6603 \cdot 10^{-27} \mathrm{~kg}-\right.$ the proton mass). The first term in the sum (1) sets the kinetic energy of the chain, the second one determines the energy of valent interactions between neighbouring particles (this term describes the chain longitudinal rigidity, the third one defines the chain bending energy, and the forth one establishes the van der Waals interactions between the atoms of the chain. The distance $r_{n}=\left|v_{n}\right|$, where the vector $v_{n}=u_{n+1}-u_{n}$, the valent angle cosine is defined as $\cos \left(\theta_{n}\right)=-\left(v_{n-1}, v_{n}\right) / r_{n-1} r_{n}$ and the distance $r_{n, k}=\left|u_{n}-u_{k}\right|$. The potential

$$
V(r)=K(r-a)^{2} / 2 \text {, }
$$

is responsible for the longitudal rigidity and the potential

$$
U(\theta)=\varepsilon[\cos (\theta)+1],
$$

sets the chain bending rigidity. The modificated LennardJones potential

$$
\begin{aligned}
& W(r)=4 \varepsilon\left[(\sigma / f(r))^{12}-(\sigma / f(r))^{6}\right], \\
& f(r)=r_{0}\left(r / r_{0}\right)^{\alpha}, \quad r_{0}=2^{1 / 6} \sigma,
\end{aligned}
$$

describes the weak van der Waals interactions of the chain nodes. The parameters of interaction potentials (2), (3), (4) are can be found in [36,37], the rigidity value $K=405 \mathrm{~N} / \mathrm{m}$, the chain step $a=0.1228 \mathrm{~nm}$, energy $\varepsilon=3.50 \mathrm{eV}, \varepsilon=0.00834$ $\mathrm{eV}$, parameters $\sigma=0.3212 \mathrm{~nm}, \alpha=0.88$.

In order to find the stationary state of the nanoribbon one should solve the minimum value problem

$$
\begin{array}{r}
E=\sum_{n=1}^{N-1} V\left(r_{n}\right)+\sum_{n=2}^{N-1} U\left(\theta_{n}\right)+\sum_{n=1}^{N-4} \sum_{k=n+4}^{N} W\left(r_{n, k}\right) \rightarrow \\
\rightarrow \min :\left\{u_{n}\right\}_{n=1}^{N},
\end{array}
$$

i.e. minimization the potential energy of the chain by its nodes coordinates $\left\{u_{n}\right\}_{\mathrm{n}=1}^{\mathrm{N}}$. Let us $\left\{u_{n}^{0}\right\}_{\mathrm{n}=1}^{\mathrm{N}}$ to consider as the solution of the minimum problem (5), then the corresponding scroll package will possess the energy $\mathrm{E}\left(u_{n}^{0}\right)$.

The minimum problem (5) has been solved numerically by means of conjugate gradient method. Specifying the initial chain nodes location followed by energy minimisation one can get different stationary packages of the nanoribbon. The eigenvalues of the matrix of second derivatives of $2 \mathrm{~N} \times 2 \mathrm{~N}$ dimensionality have been determined in order to verify the stability of the established stationary package.

$$
B=\left(\left.\frac{\partial^{2} E}{\partial u_{n} \partial u_{k}}\right|_{\left\{u_{i}^{0}\right\}_{i=1}^{N}}\right)_{n=1, k=1}^{N, N}
$$

The stationary package of the chain is stable in case if all the eigenvalues of the symmetrical matrix $B$ are non-negative $\lambda_{i} \geq 0, i=1,2, \ldots, 2 N$. Three first values are always equal to zero $\lambda_{1}=\lambda_{2}=\lambda_{3}=0$. This eigenvalues correspond to the movement of the chain in the plain as a rigid body (corresponding to shear strain by two coordinates and rotation). The remaining positive eigenvalues $\lambda_{i}>0$ correspond to the natural oscillations of the structure with frequencies $\omega_{i}=\sqrt{\lambda_{3+i} / M}, i=1,2, \ldots, 2 N-3$.

When the length of the scrolled nanoribbons exceeds $L>13.39$ spiral chain configuration has been revealed to be the most energetically favourable [36]. The cross-section of the scroll package can be described as a truncated Archimedean spiral - see Fig. 1 (b). The helical pitch (shortest distance between atoms of adjacent layers) makes $h=0.34 \mathrm{~nm}$. The spiral (scroll) can be characterized by a number of coils $N_{c}$. A blank hollow always present in the centre of the spiral makes it similar to a circular hollow with the diameter $2 R_{2}$ (where $R_{2}$ is the mean radius of the outer spiral coil) and with an inner radius of the hole $R_{1}$ (the average radius of the inner spiral coil). With the increase in the scrolled nanoribbon length $L$ number of coils grows proportionally to $N_{c} \approx 0.33 L^{0.63}$ increases as, and its radii growth can me described by the dependence $R_{1} \approx 0.67 L^{0.2}, R_{2} \approx 0.4 L^{0.47}$ (all dimensions in nanometers) - see Fig. 2.

Typical low frequency vibrations of the scroll are shown on Fig. 3 and Fig.4. The natural vibration of the scroll with the lowest frequency $\omega_{1}$ corresponds to the softest mode of the scrolled package oscillation in "twisting - untwisting" mode. The cross section of the scroll moves periodically following the path of Archimedes spiral shape forward and back to its centre (Fig. 3), and the number of coils is changed periodically (Fig. 5). The oscillations frequency drops monotonically as a reverse function of the nanoribbons length $\omega_{1} \approx 36 / L$, where $\mathrm{L} \rightarrow \infty$. Similar asymptotic behaviour has been obtained analytically by means of the continuum model of an elastic spiralled rod [21, 34].

Following lowest frequencies $\omega_{2}, \omega_{3}$ correspond to natural vibrations in the mode of periodical transverse spiral compressions - see Fig. 4.

Weak distinct of the spiral shape from a perfectly round hollow leads to a small difference between these frequencies (see. Fig. 2, curves 4 and 5). Spiral compressions corresponding to these frequencies occur along mutually orthogonal directions. Increase of the nanoribbon length promotes the tendency of frequencies decrease and getting close to zero at a slower rate $\omega_{2,3} \approx 30 / L^{0.65}$, at $\mathrm{L} \rightarrow \infty$.

\section{High amplitude natural vibrations}

The analysis of the eigenvalues and corresponding matrix (6) eigenvectors allows us to describe only low-amplitude (harmonic) scroll natural oscillations. In order to make the analysis of high-amplitude oscillations the initiation of those oscillations with different initial impulse energy should be performed.

Let us consider a chain containing $N=400$ nodes (nanoribbon length $L=49.00 \mathrm{~nm}$ ). The corresponding stationary scroll is shown in Fig. 3 (b). Small-amplitude natural scroll oscillations are characterized by frequencies 

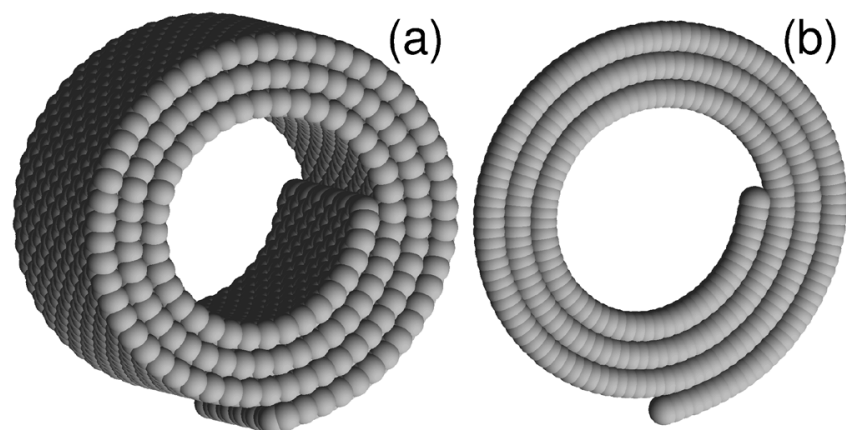

Fig. 1. Three dimensional full-atomic model of the nanoribbon (a) and corresponding two dimensional chain model (b). The nanoribbon length $L x=36.84 \mathrm{~nm}$ and width $L z=2.55 \mathrm{~nm}$ (number of nodes $N=301, K=6$, number of atoms $N_{\text {oll }}=2 N K=3612$ ), number of coils $N_{c}=3.27$, internal scroll radius $R_{1}=1.431 \mathrm{~nm}$, external $R_{2}=2.184 \mathrm{~nm}$
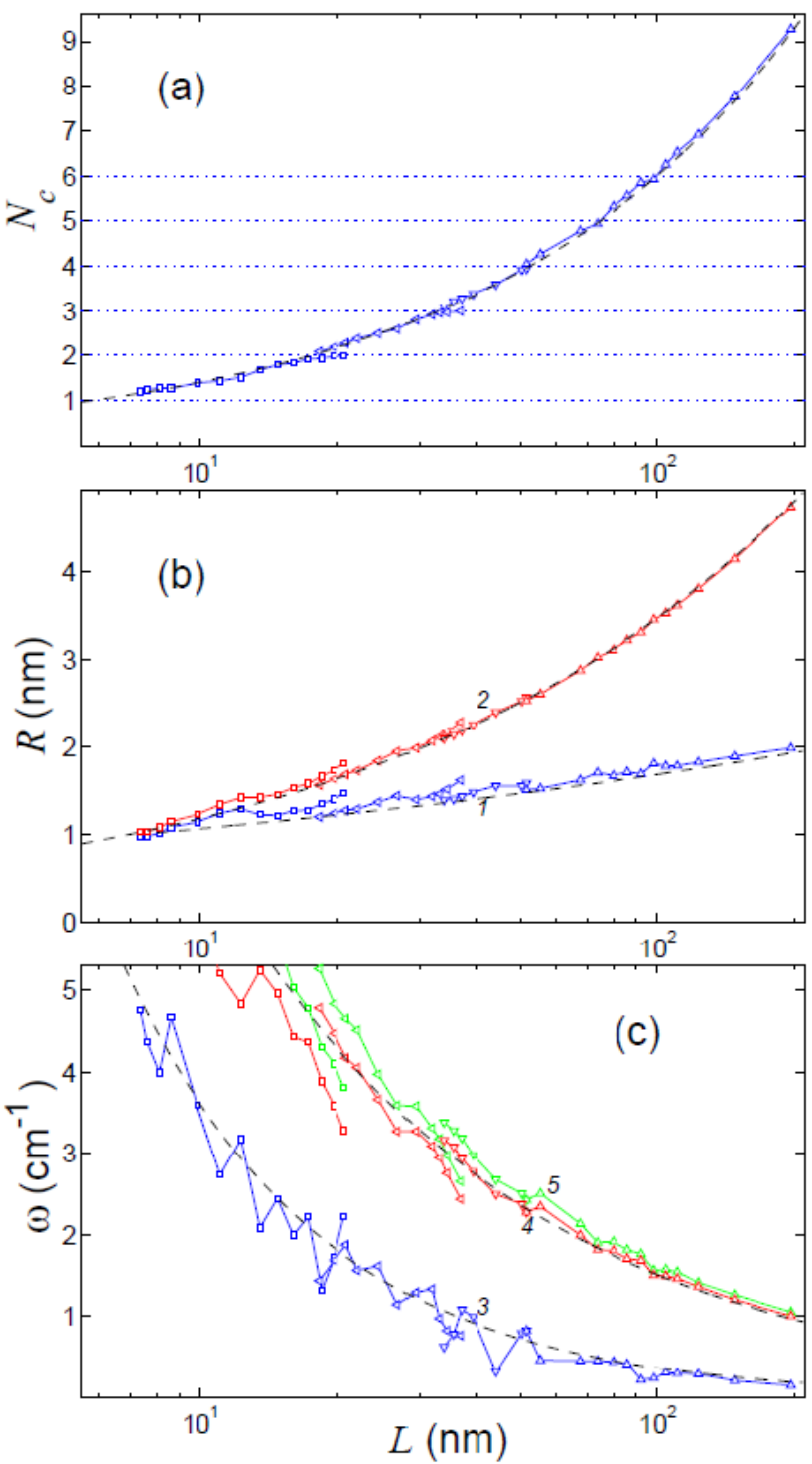

Fig. 2. Effect of the nanoribbon length $L$ on (a) the number of coils of the stationary scroll $N_{\text {c }}$, (b) inner $R_{1}$ and outer $R_{2}$ radii of the scroll (curves 1 and 2), (c) the lowest natural vibration frequencies of the scroll $\omega_{1}, \omega_{2}$ and $\omega_{3}$. Dashed lines stand for dependencies $N_{c}=0.33 L^{0.63}, R_{1}=0.67 L^{0.2}, R_{2}=0.4 L^{0.47}, \omega_{1}=36 L^{-1}$ and $\omega_{2}=30 L^{-0.65}$.
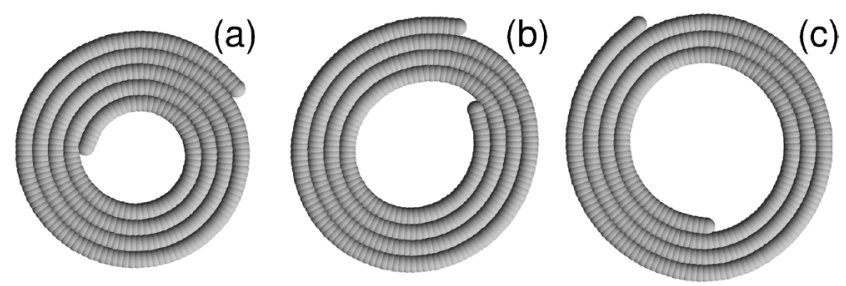

Fig. 3. Maximal twist (a), basic state (b) and maximal untwist of the scrolled nanoribbon with length $L=49.00 \mathrm{~nm}$ (number of chain nodes $N=400$ ) at its natural vibration in "twisting - untwisting" mode. The value of oscillation energy is equal to $E_{0}=0.16 \mathrm{eV}$, the oscillation period makes $T=123.2$ ps (frequency $\omega=0.27 \mathrm{~cm}^{-1}$ ).
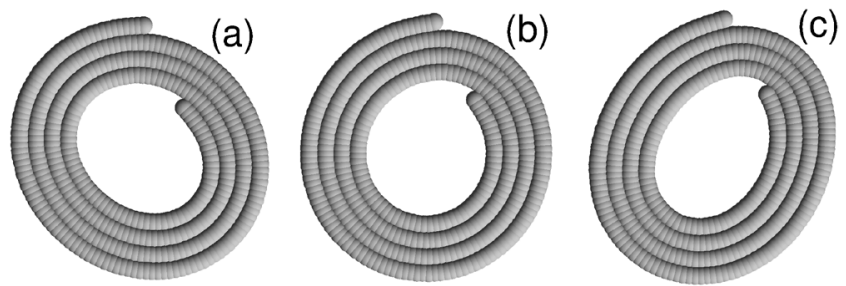

Fig. 4. Maximal lateral compression (a), basic state (b) and maximal strain of the scrolled nanoribbon with length $L=49.00 \mathrm{~nm}$ (number of chain nodes $N=400$ ) involved in its natural vibration in "strain - compression" mode. The value of oscillation energy $E_{0}=0.1 \mathrm{eV}$, the oscillation period $T=13.4 \mathrm{ps}$ (frequency $\omega=2.49 \mathrm{~cm}^{-1}$ ).
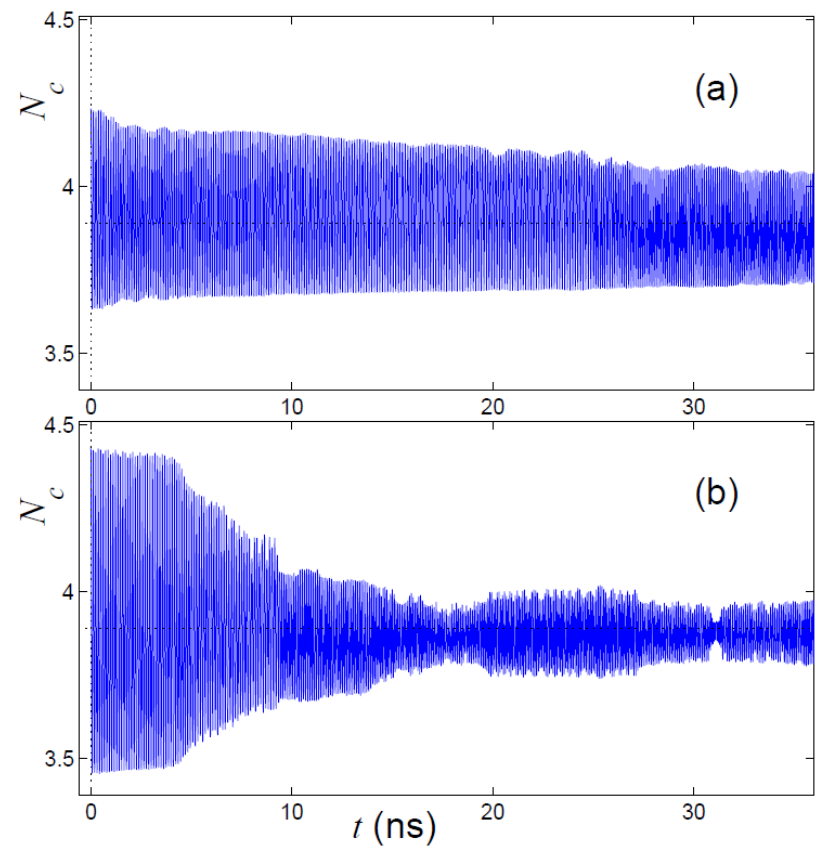

Fig. 5. Dependence of coil number $N_{c}$ on the time $t$ in case of excitation of natural vibration in "twisting - untwisting" mode with energy $E_{0}=0.07 \mathrm{eV}$ (a) and $0.16 \mathrm{eV}$ (b). The nanoribbon length $L=49.00 \mathrm{~nm}$ (number of chain nodes $N=400$ ). 
$\omega_{i}=0.34,2.45,2.58,6.90,7.17,9.13, \ldots \mathrm{cm}^{-1}$. Knowing the corresponding eigenvectors, we can excite corresponding vibrations. We also can change the initial excitement energy by changing the eigenvector amplitude.

Numerical simulation of the nanoribbon dynamics revealed that oscillations corresponding to the three lowest natural frequencies $\omega_{1}, \omega_{2}$ and $\omega_{3}$ appeared to be soft anharmonic vibrations characterized by the increase of amplitude as the oscillation frequency decreases. One should mention that there exists a critical value of energy promoting the interaction of these modes.

The natural vibration "twisting-untwisting" mode remains stable upon the energy level $E_{0}=0.1 \mathrm{eV}$. It is accompanied by a slow exponential decrease of oscillations amplitude - see Fig. 5 (a). This oscillation mode does not interact with other scroll natural vibrations - see Fig. 6 (a). At higher energy values the oscillation becomes unstable and the oscillations amplitude decreases sharply - see Fig. 5 (b).

Primarily the oscillations with frequencies being the multiples of the fundamental frequency are emerged. Next turns corresponds to emerging of transverse scroll compression vibrations with frequencies $\omega_{2}, \omega_{3}-$ see Fig. 6 (b).

Thus, a weak anharmonicity of "twisting-untwisting" vibration mode leads to a decrease of the oscillations frequency (amplitude increase results in frequency change from initial value $\omega_{1}=0.34 \mathrm{~cm}^{-1}$ to minimal frequency $0.27 \mathrm{~cm}^{-1}$ ). The strong anharmonicity emerges the energy transfer to the high frequency natural vibration of the scroll transverse compression (frequencies $\omega_{2,3}=2.45,2.58 \mathrm{~cm}^{-1}$ ).

The transverse-compression scroll oscillations also remains stable with energy $E_{0} \leq 0.1 \mathrm{eV}$, and it is accompanied by a very slow transfer of energy to the lowest -frequency "twisting-untwisting" vibration mode - see Fig. 7 (a). Higher energy states are characterized by a rapid energy transfer to the low frequency natural vibration without excitation of high frequency vibrations - see Fig. 7 (b).

\section{Conclusions}

The simulation of the natural oscillations of the scrolled carbon nanoribbons revealed that the first three lowest frequency oscillations ("twisting-untwisting" and "transverse-compression" vibration modes of the scroll) are stable only in case if their energy does not exceed the value $0.1 \mathrm{eV}$. In this case, the vibrations anharmonicity results solely in frequency reduction. At higher energies the anharmonicity driven interaction of these three lowfrequency modes is emerged and excitation of one mode leads to initiation of the two others.

Acknowledgements. A.V. Savin thanks financial support provided by the Russian Science Foundation, grant N 14-1300982. E.A. Korznikova is grateful for the financial support from the President Grant for young scientists (grant N MK5283.2015.2). S.V. Dmitriev appreciates the support from the Tomsk State University Academic D.I. Mendeleev Fund Program and grant RFBR 14-02-97029. Calculations have been performed of computer facilities of Joint Supercomputer Centre of the Russian Academy of Sciences.

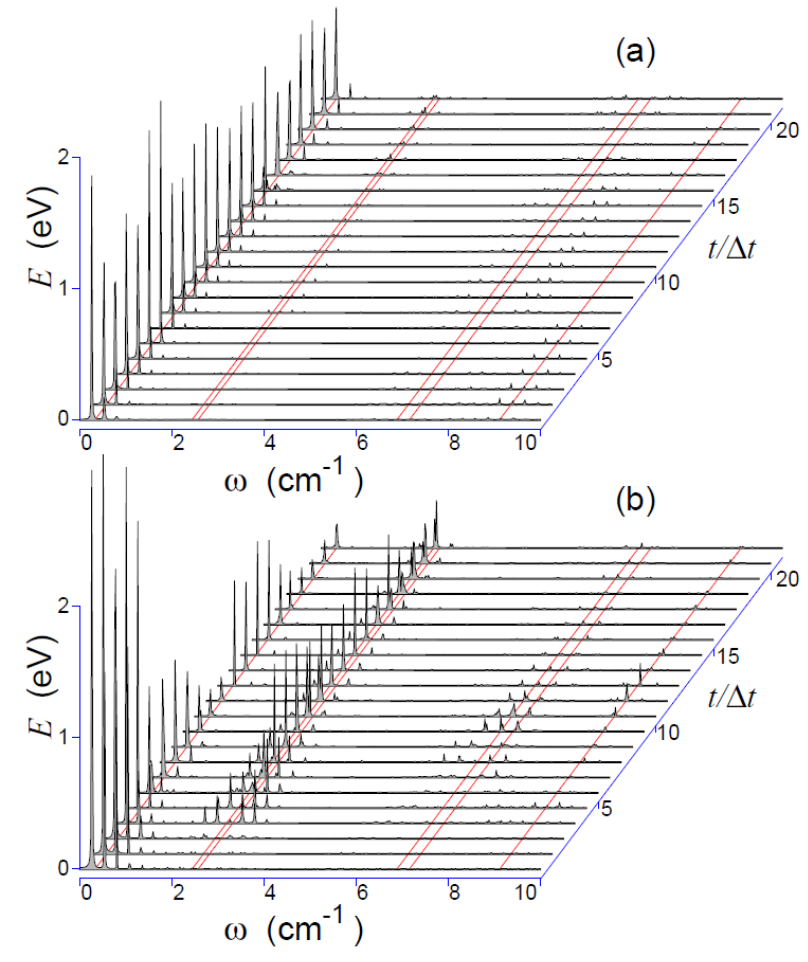

Fig. 6. The time dependence of the frequency of the scroll kinetic energy distribution after excitation its natural vibration in "twisting - untwisting" mode with energy $E_{0}=0.07 \mathrm{eV}$ (а) и $0.16 \mathrm{eV}$ (b). The length of the nanoribbon makes $L=49.00 \mathrm{~nm}$ (the number of chain nodes $N=400$ ), the time step equal to $\Delta t=1.6384 \mathrm{~ns}$. Straight lines indicate the frequencies of the low-amplitude natural scroll oscillations $\omega_{i}=0.34,2.45,2.58,6.90,7.17,9.13 \mathrm{~cm}^{-1}$.

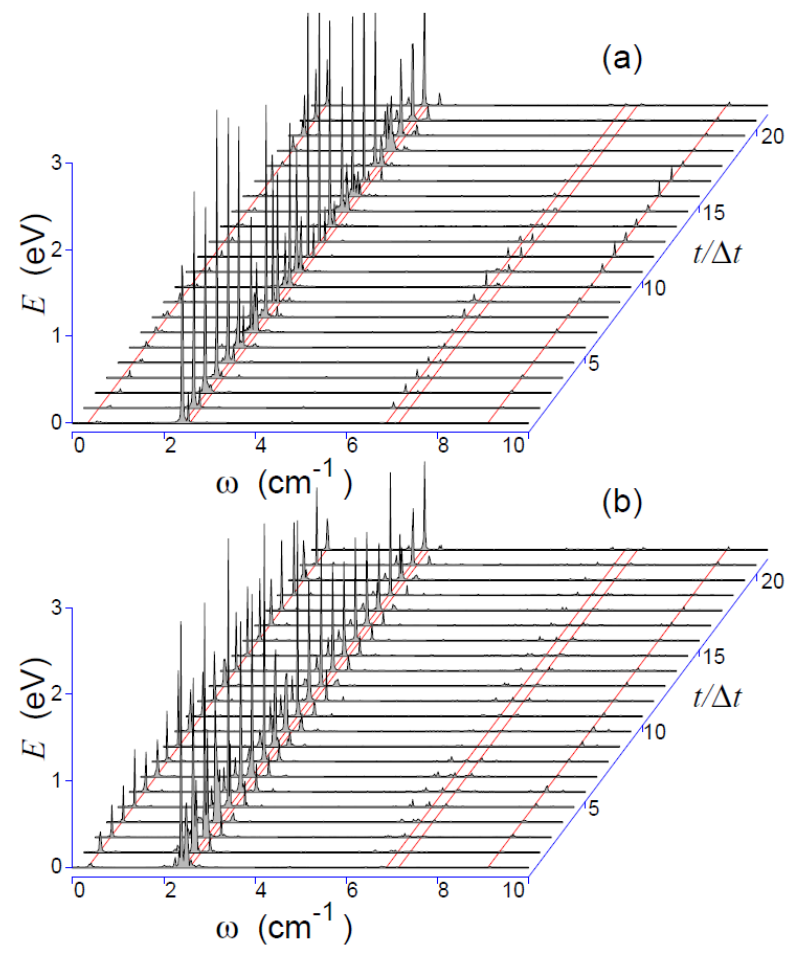

Fig. 7. The time dependence of the frequency of the scroll kinetic energy distribution after excitation its natural vibration in "strain - compression" mode with energy $E_{0}=0.1 \mathrm{eV}$ (а) и $0.15 \mathrm{eV}$ (b). The length of the nanoribbon makes $L=49.00 \mathrm{~nm}$ (the number of chain nodes $N=400$ ), the time step equal to $\Delta t=1.6384 \mathrm{~ns}$. Straight lines indicate the frequencies of the low-amplitude natural scroll oscillations $\omega_{i}=0.34,2.45,2.58,6.90,7.17,9.13 \mathrm{~cm}^{-1}$. 


\section{References}

1. K. S. Novoselov, A. K. Geim, S. V. Morozov, D. Jiang, Y. Zhang, S. V. Dubonos, I. V. Grigorieva, and A. A. Firsov, Science 306, 666 (2004).

2. A. K. Geim and K. S. Novoselov, Nat. Mater. 6, 183 (2007).

3. C. Soldano, A. Mahmood, and E. Dujardin, Carbon 48, 2127 (2010).

4. J. A. Baimova, B. Liu, S. V. Dmitriev, and K. Zhou, Phys. Status Solidi - R 8, 336 (2014).

5. J. A. Baimova, B. Liu, S. V. Dmitriev, N. Srikanth, K. Zhou, Phys. Chem. Chem. Phys. 16, 19505 (2014).

6. J.A. Baimova, E. A. Korznikova, S. V. Dmitriev, B. Liu, K. Zhou, Rev. Adv. Mater. Sci. 39, 69 (2014).

7. A. V. Orlov and I. A. Ovid'ko, Rev. Adv. Mater. Sci. 40, 249 (2015).

8. Z. Xu and M. J. Buehler, ACS Nano 4, 3869 (2010).

9. W. Li, X. Zheng, B. Liu, X. Sun, T. Wang, J. Zhang, Y. Yan, Carbon 89, 272 (2015).

10. W. Bollmann and J. Spreadborough, Nature 186, 29 (1960).

11. S. Zhu and T. Li, J. Phys. D: Appl. Phys. 46, 075301 (2013).

12. G. Cheng, I. Calizo, X. Liang, B.A. Sperling, A.C. Johnston-Peck, W. Li, J.E. Maslar, C.A. Richtera, and A. R. H. Walker, Carbon 76, 257 (2014).

13. H. Q. Zhou, C. Y. Qiu, H. C. Yang, F. Yu, M. J. Chen, L. J. Hu, Y. J. Guo, and L. F. Sun, Chem. Phys. Lett. 501, 475 (2011).

14. X. Chen, R.A. Boulos, J.F. Dobson, and C.L. Raston, Nanoscale, 5, 498 (2013).

15. M.V. Savoskin, V.N. Mochalin, A.P. Yaroshenko, N.I. Lazareva, T.E. Konstantinova, I. V. Barsukov, and I. G. Prokofiev, Carbon 45, 2797 (2007).

16. X. Xie, L. Ju, X. Feng, Y. Sun, R. Zhou, K. Liu, S. Fan, Q. Li, and K. Jiang, Nano Lett. 9, 2565 (2009).

17. A.L. Chuvilin, V.L. Kuznetsov, and A.N. Obraztsov, Carbon 47, 3099 (2009).
18. H. Pan, Y. Feng, and J. Lin, Phys. Rev. B 72, 085415 (2005).

19. R. Rurali, V. R. Coluci, and D.S. Galvao, Phys. Rev. B 74, 085414 (2006).

20. Y. Chen, J. Lu, and Z. Gao, J. Phys. Chem. C 111, 1625 (2007).

21. X. Shi, N. M. Pugno, Y. Cheng, and H. Gao, J. Appl. Phys. 95, 163113 (2009).

22. B. V. C. Martins and D.S. Galvao, Nanotechnology 21, 075710 (2010).

23. S. Huang, B. Wang, M. Feng, X. Xu, X. Cao, and Y. Wang, Surf. Sci. 634, 3 (2015).

24. E. Perim, R. Paupitz, and D. S. Galvao, J. Appl. Phys. 113 , 054306 (2013).

25. Y. Wang, H.F. Zhan, C. Yang, Y. Xiang, and Y. Y. Zhang, Comp. Mater. Sci 96300 (2015).

26. X. Shi, Y. Cheng, N. M. Pugno, and H. Gao, J. Appl. Phys. 96, 053115 (2010).

27. Z. Zhang and T. Li, Appl. Phys. Lett. 97, 081909 (2010).

28. L. Chu, Q. Xue, T. Zhang, and C. Ling, J. Phys. Chem. C 115, 15217 (2011).

29. N. Patra, Y. Song, and P. Kral, ACS Nano 5, 1798 (2011).

30. H. Y. Song, S. F. Geng, M. R. An, and X. W. Zha, J. Appl. Phys. 113, 164305 (2013).

31. X. Shi, Y. Cheng, N.M. Pugno, H. Gao Small, 6 (6), 739 744 (2010).

32. L. J. Yi, Y. Y. Zhang, C. M. Wang, and T. C. Chang, J. Appl. Phys. 115, 204307 (2014). Nanoscale 5, 5450 (2013).

33. Z. Zhang, Y. Huang, and T. Li, J. Appl. Phys. 112, 063515 (2012).

34. X. Shi, N. M. Pugno, H. Gao, Acta Mech. Solida Sin. 23, $484(2010)$

35. X. Shi, N. M. Pugno, H. Gao, Int. J. Fract. 171, 163 (2011).

36. A.V. Savin, E.A. Korznikova, and S.V. Dmitriev, Phys. Rev. B 92, 035412 (2015).

37. A. V. Savin, E. A. Korznikova, S. V. Dmitriev, Physics of the Solid State 57(11), 2348-2355 (2015). 